\title{
Pancreatobiliary Disorders Associated with Pancreaticobiliary Maljunction
}

\author{
Terumi Kamisawa $^{a}$ Goro Honda $^{b}$ Masanao Kurata ${ }^{b}$ Michiyo Tokura ${ }^{b}$ \\ Koji Tsurutab \\ Departments of a Internal Medicine and ${ }^{\mathrm{b}}$ Surgery, Tokyo Metropolitan Komagome Hospital, Tokyo, Japan
}

\begin{abstract}
Key Words
Acute pancreatitis - Bile duct carcinoma $\cdot$ Chronic pancreatitis - Gallbladder carcinoma • Pancreaticobiliary maljunction
\end{abstract}

\begin{abstract}
Aim: To clarify the features of biliary carcinomas and pancreatic disorders associated with pancreaticobiliary maljunction (PBM). Method: A total of 107 PBM patients with biliary dilatation were reviewed ( 52 with and 55 without PBM). Clinicopathological findings of biliary carcinomas, and acute or chronic pancreatitis were examined in association with PBM. We also investigated pancreatographic findings in PBM patients and the relationship to their clinical findings in view of pancreatic juice flow. Results: Gallbladder and bile duct carcinomas were seen in 11 (21\%) and 8 (15\%) of the 52 patients with PBM and biliary dilatation, respectively. In patients with PBM without biliary dilatation $(n=55)$, only gallbladder carcinoma was detected in 36 (65\%) patients. At diagnosis, patients with gallbladder or bile duct carcinoma associated with PBM were younger than those without PBM $(p<0.01)$. Gallstones were detected only in $6 \%$ of the patients with PBM-associated gallbladder carcinoma. Multiple biliary carcinomas were detected in 3 patients (38\%) with PBM-associated bile duct carcinoma. While 48 patients (60\%) with biliary carcinoma and PBM had a normal pancreatic duct system, only 2 gallbladder carcinomas occurred in 11 dorsal pancreatic duct (DPD)-dominant patients ( $p<0.05$ ). Amy-
\end{abstract}

lase concentration in the bile of DPD-dominant patients was significantly lower than that of patients with a normal pancreatic duct system $(p<0.05)$. Ten PBM patients had pancreatic disorders: acute pancreatitis $(n=3)$, chronic pancreatitis $(n=5)$, and pancreatic carcinoma $(n=2)$. Conclusions: In PBM patients, pancreatobiliary reflux frequently leads to biliary cancer, and biliopancreatic reflux often leads to acute or chronic pancreatitis. The biliary tract of PBM patients can be considered premalignant.

Copyright $\odot 2010$ S. Karger AG, Basel

\section{Introduction}

Pancreaticobiliary maljunction (PBM) is a congenital anomaly defined as the union of the pancreatic and biliary ducts outside of the duodenal wall. As the action of the sphincter muscle does not functionally affect the union, two-way regurgitation (pancreatobiliary and biliopancreatic reflux) occurs, resulting in various pathological conditions in the biliary tract and in the pancreas [1-5]. Given that the hydropressure within the pancreatic duct is usually greater than in the bile duct [6], pancreatic juice frequently refluxes into the bile duct via the anomalous junction, resulting in a high incidence of carcinogenesis in the biliary tract. Although congenital choledochal cysts are usually accompanied by PBM, some patients with PBM show no dilatation of the extrahepatic bile duct. In this study, we clarified the features of biliary

\section{KARGER \\ Fax +41613061234 \\ E-Mail karger@karger.ch}

www.karger.com
(C) 2010 S. Karger AG, Basel

$0253-4886 / 10 / 0272-0100 \$ 26.00 / 0$

Accessible online at:

www.karger.com/dsu
Terumi Kamisawa, MD, PhD

Department of Internal Medicine, Tokyo Metropolitan Komagome Hospital

3-18-22 Honkomagome, Bunkyo-ku

Tokyo 113-8677 (Japan)

Tel. +81 33823 2101, Fax +8133824 1552, E-Mail kamisawa @ cick.jp 
carcinoma and pancreatic disorders associated with PBM. Furthermore, we investigated the pancreatographic findings in PBM patients and the relationship to their clinical findings in view of pancreatic juice flow.

\section{Patients and Methods}

We treated 107 patients with PBM from 1973 to 2009. There were 27 men and 80 women with a mean age of 49.0 years (range 8-84 years). PBM was diagnosed by endoscopic retrograde cholangiopancreatographic or percutaneous transhepatic cholangiographic evidence of a lack of effect of the sphincter of Oddi at the union of the pancreatic and biliary ducts, and the presence of a long common channel (>15 mm). The PBM group was divided into two subgroups: those with biliary dilatation (maximal bile duct diameter $>10 \mathrm{~mm} ; \mathrm{n}=52$ ) and those without biliary dilatation $(\mathrm{n}=55)$.

Clinicopathological findings of biliary carcinomas and pancreatic disorders associated with PBM were examined, comparing 232 cases of gallbladder carcinoma with 159 cases of bile duct carcinoma which were not associated with PBM.

We reviewed the cholangiopancreatograms, and visualization was satisfactory in $91 \mathrm{PBM}$ patients. The maximum diameters of both the ventral pancreatic duct and Santorini's duct were measured using a manual goniometer. When the maximum diameter of Santorini's duct was almost equal to or greater than that of the ventral pancreatic duct, the relationship between both ducts was defined as dorsal pancreatic duct (DPD) dominance (fig. 1) [7, 8]. Amylase level in the bile was measured in 19 PBM patients.

For statistical analyses, Fisher's exact probability test and the Mann-Whitney $U$ test were employed, and $\mathrm{p}<0.05$ was considered significant.

\section{Results}

\section{Clinicopathological Features of Gallbladder and Bile}

Duct Carcinoma Associated with PBM

In $52 \mathrm{PBM}$ patients with biliary dilatation, gallbladder and bile duct carcinoma were seen in $11(21 \%)$ and $8(15 \%)$ patients, respectively. In 55 PBM patients without biliary dilatation, only gallbladder carcinoma was detected in 36 (65\%) patients. At diagnosis, patients with PBM-associated gallbladder carcinoma were significantly younger (mean \pm SD: $58.5 \pm 8.6$ ) than those with gallbladder carcinoma without PBM (69.4 \pm 8.7; p <0.01). Gallstones were detected only in $6 \%$ of the patients with PBM-associated gallbladder carcinoma, being significantly less frequent than in those with gallbladder carcinoma without PBM (62\%; $\mathrm{p}<0.01$; table 1).

The 8 patients with PBM-associated bile duct carcinoma were significantly younger $(54.6 \pm 14.2$ years) than those with gallbladder carcinoma without PBM at diag-

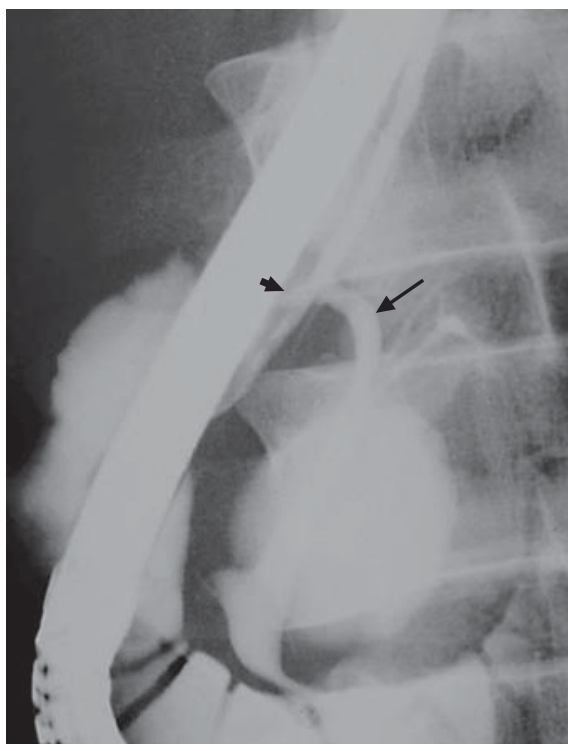

Fig. 1. Retrograde cholangiopancreatogram of a DPD-dominant patient with PBM and bile duct dilatation. The end of the long ventral pancreatic duct (long arrow) fused with the dorsal pancreatic duct (arrowhead).

nosis $(68.8 \pm 9.1 ; \mathrm{p}<0.01)$. Multiple biliary carcinomas ( 2 multiple bile duct carcinomas and 1 carcinoma of the bile duct and gallbladder) were detected in 3 patients (38\%) with PBM-associated bile duct carcinoma compared to only $4 \%$ in those with bile duct carcinoma without PBM ( $\mathrm{p}<0.01$; table 1$)$. Clinicopathological features of papillary adenocarcinomas $(n=4)$ differed from those of tubular adenocarcinomas $(n=4)$. Radiologically, papillary adenocarcinoma presented as an eccentrically located polypoid mass in the cyst, while there was evidence of bile duct stenosis with irregular thickening of the bile duct wall in tubular adenocarcinoma. Papillary adenocarcinoma was associated with extensive superficial spread $(\mathrm{n}=1)$, and synchronous $(\mathrm{n}=2)$ or metachronous $(\mathrm{n}=1)$ multicentric tumors. Due to extensive or repeated resection, 2 patients survived $>2$ years.

\section{PBM-Associated Biliary Carcinoma Showing DPD}

Dominance or a Normal Pancreatic Duct System

Pancreatographic findings were divided into a normal duct group (80 patients) and a DPD-dominant group (11 patients; fig. 1). Partial pancreas divisum was noted in 6 of $11 \mathrm{DPD}$-dominant patients. While 48 patients (60\%) with biliary carcinoma ( 42 with gallbladder carcinoma and 6 with bile duct carcinoma) had PBM with a normal 
Table 1. Clinicopathological features of gallbladder and bile duct carcinoma associated with or without PBM

\begin{tabular}{|c|c|c|c|c|}
\hline & \multicolumn{2}{|c|}{ Gallbladder carcinoma } & \multicolumn{2}{|c|}{ Bile duct carcinoma } \\
\hline & $\begin{array}{l}\text { with PBM } \\
(\mathrm{n}=47)\end{array}$ & $\begin{array}{l}\text { without PBM } \\
(\mathrm{n}=232)\end{array}$ & $\begin{array}{l}\text { with PBM } \\
(\mathrm{n}=8)\end{array}$ & $\begin{array}{l}\text { without PBM } \\
(\mathrm{n}=159)\end{array}$ \\
\hline Age at diagnosis, years & $58.5 \pm 8.6^{*}$ & $69.4 \pm 8.7$ & $54.6 \pm 14.2$ & $68.8 \pm 9.1$ \\
\hline Male:female ratio & $1: 3.3$ & $1: 2.9$ & $2: 6$ & $1: 0.7$ \\
\hline Association with gall stones & $3 / 47(6 \%)^{*}$ & $140 / 226(62 \%)$ & & \\
\hline Multiple biliary carcinoma & $4 / 47(9 \%)$ & $11 / 232(5 \%)$ & $3 / 8(38 \%)^{*}$ & $6 / 159(4 \%)$ \\
\hline Multiple gallbladder carcinoma & 3 & 5 & 2 & 0 \\
\hline Combined carcinoma of gallbladder and bile duct & 1 & 6 & 1 & 6 \\
\hline
\end{tabular}

pancreatic duct system, only 2 gallbladder carcinomas $(18 \%)$ occurred in DPD-dominant patients $(\mathrm{p}<0.05)$.

Although there was no difference in the diameter of the ventral pancreatic duct, the maximum diameter of the Santorini duct in DPD-dominant patients was significantly larger than that in patients with a normal pancreatic duct system $(2.5 \pm 0.6$ vs. $0.9 \pm 0.3, p<0.01)$. The Santorini duct arose directly from the upstream DPD in DPD-dominant patients. Biliary amylase concentration was significantly lower in DPD-dominant patients than in patients with a normal pancreatic duct system $(75,403.5$ $\pm 82,015.4$ vs. $278,157.0 \pm 207,395.0$ IU/l; $\mathrm{p}<0.05$ ).

\section{Pancreatic Disorders Associated with PBM}

Ten PBM patients had pancreatic disorders: acute pancreatitis $(n=3)$, chronic pancreatitis $(n=5)$, and pancreatic carcinoma $(\mathrm{n}=2)$. Acute pancreatitis was mild $(\mathrm{n}=$ $3)$ and relapsing $(n=2)$. In patients with chronic pancreatitis, pancreatic stones $(n=2)$ and radiolucent protein plugs $(n=2)$ were detected only in the dilated common channel or in the main pancreatic duct near the common channel. Two patients treated with cystoduodenostomy during infancy developed chronic pancreatitis 11 and 27 years later, respectively.

\section{Discussion}

Gallbladder carcinoma was detected in $44 \%$ of the patients with PBM. Their age at diagnosis was significantly younger ( $\sim 10$ years) than in those with gallbladder carcinoma without PBM. Gallstones were rarely detected in patients with PBM-associated gallbladder carcinoma, although they were usually detected in gallbladder carci- noma without PBM. Bile duct carcinoma was detected in $8 \mathrm{PBM}$ patients with biliary dilatation. In patients with PBM-associated bile duct carcinoma, age was significantly lower (14 years) at diagnosis than in those with gallbladder carcinoma without PBM. Furthermore, multiple biliary carcinomas were a frequent finding in the patients with PBM. These findings suggest that the biliary tract of patients with PBM has a carcinogenic potential which differs from the tumorigenic mechanisms resulting in biliary carcinomas in patients without PBM [9-11].

Only gallbladder carcinoma was detected in $65 \%$ of PBM patients without biliary dilatation. The mechanism of carcinogenesis in PBM is considered to be related to stasis of bile combined with reflux of pancreatic juice via a long common channel [12]. Aoki et al. [13] reported that amylase levels were lower in the bile duct of patients with PBM without biliary dilatation, and postoperative scintigraphy of their biliary system revealed no evidence of cholestasis. Bile stasis appears to be less severe in PBM patients without biliary dilatation. Treatment for PBM with biliary dilatation is prophylactic flow diversion surgery with complete resection of the dilated common bile duct. We remove the dilated extrahepatic bile duct and perform reconstruction of the biliary tract. To completely resect the dilated common bile duct, we excise the common bile duct from the pancreas to the narrow segment toward the junction. The narrow segment can be safely circumvented by exposing the outer plane of the epicholedochal plexus. This plexus is an inner part of the subserosal layer, at which reticulated thin vessels are observed on the exposed plane. We expose this plane, sealing and cutting thin vessels that supply this plexus using bipolar cautery. Then, on the hepatic side, we aim to remove the dilated common bile duct completely. To con- 
firm the conformation of the confluence of hepatic ducts and determine an adequate transection level of the common bile duct, we open the anterior wall of the common bile duct and observe the orifices of the hepatic ducts from the interior. In patients with PBM accompanied by dilatation of the intrahepatic bile duct, there are usually stenotic portions of the hepatic duct on the hepatic side of the dilated common bile duct. If such a stenotic portion remains after reconstruction of the biliary tract, it often causes intrahepatic cholestasis, cholangitis, or intrahepatic calculi. Therefore, we remove such a stenotic portion along with the dilated common bile duct, if possible. If the stenotic portion cannot be removed or if we detect a membranous septum or a slender column of the intrahepatic bile duct within the lumen, we ablate these under direct inspection, or we create a wide stoma by an incision along the lateral wall of the hepatic ducts over the relatively stenotic portions. Then, we basically select hepaticojejunostomy (Roux-en-Y) to reconstruct the biliary tract. Especially after ablation, we mark a point to place an indwelling silicone stent through that portion to prevent recurrence of stenosis. If we cannot manage the stenotic portion from the hepatic hilum, we consider hepatectomy; however, when we are obliged to dilate the stenotic portion with a balloon due to the patient's condition or other factors, we place an indwelling silicone stent through that portion just as we would after ablation.

However, consensus has not been reached on whether preventive resection of the extrahepatic bile duct is necessary in PBM patients without dilatation of the extrahepatic bile duct [14]. In our institute, only laparoscopic cholecystectomy is performed as prophylactic treatment for PBM patients without biliary dilatation, and no bile duct carcinoma has developed in these patients during the postoperative follow-up.

As development progresses embryologically, the duct of the dorsal bud usually undergoes a certain degree of atrophy at its duodenal end and becomes a smaller pancreatic duct $[15,16]$. Although Santorini's duct dominance, in which the ventral pancreatic duct is narrower and anastomoses with Santorini's duct, is observed in about $4 \%$ of pancreases $[17,18]$, DPD dominance was detected in $12 \%$ of PBM patients. In the present study, biliary carcinoma was significantly less frequent in DPDdominant patients with PBM than in those with a normal pancreatic duct system. Furthermore, lower biliary amylase levels were more frequently seen in DPD-dominant patients. Consequently, it is suggested that in PMB patients with DPD dominance most pancreatic juice in the upper DPD is drained into the duodenum via the minor duodenal papilla, thus decreasing reflux of pancreatic juice to the biliary tract and resulting in a lower frequency of associated biliary carcinoma.

The incidence of acute pancreatitis in adults with a choledochal cyst is reported to range from 18 [19] to $23 \%$ [20]. In the present study, 2 of 3 PBM patients developing acute pancreatitis had a choledochal cyst. Raffensperger et al. [21] postulated that bile reflux into the pancreatic duct is the most likely cause of pancreatitis. Kaneko et al. [22] reported that radiolucent filling defects consisting of protein plugs were detected in the common channel in $40 \%$ of patients with a choledochal cyst $(n=559)$, and the protein plugs disappeared spontaneously or after irrigation in $82 \%$ of them. It is supposed that during an acute pancreatitis attack in PBM patients, a transient increase in the intraductal pressure occurs due to bile reflux into the pancreatic duct, which may be induced when a long common channel is temporarily obstructed for various reasons, including protein plugs or dysfunction of the sphincter of Oddi.

Cholangiopancreatographic characteristics of chronic pancreatitis with PBM were filling defects occurring only in the dilated common channel or in the main pancreatic duct near the common channel. They were sometimes transparent on X-ray films and consisted mostly of protein. Two patients treated with cystoduodenostomy during infancy developed chronic pancreatitis 11 and 27 years later, respectively. Pancreatic secretory stasis or pancreatic juice mixed with bile in the dilated common channel might account for the formation of pancreatic stones or protein plugs.

In PBM patients, pancreatobiliary reflux frequently leads to biliary cancer, and biliopancreatic reflux often leads to acute or chronic pancreatitis. The biliary tract of PBM patients can be considered premalignant, and prophylactic surgery is recommended as soon as PBM is diagnosed.

\footnotetext{
References $\quad D_{1}$ The Japanese Study Group on Pancreaticobiliary Maljunction: Diagnostic criteria of pancreaticobiliary maljunction. J Hepatobiliary Pancreat Surg 1994;1:219-221.

2 Kamisawa T, Takuma K, Anjiki H, Egawa N, Kurata M, Honda G, Tsuruta K, Sasaki T: Pancreaticobiliary maljunction. Clin Gastroenterol Hepatol 2009;7:S84-S88.

-3 Kamisawa T, Anjiki H, Egawa N, Kurata M, Honda G, Tsuruta K: Diagnosis and clinical implications of pancreatobiliary reflux. World J Gastroenterol 2008;14:6622-6626.
} 
-4 Kamisawa T, Kurata M, Honda G, Tsuruta K, Okamoto A: Biliopancreatic reflux - pathophysiology and clinical implications. J Hepatobiliary Pancreat Surg 2009;16:19-24.

5 Kamisawa T, Okamoto A: Biliopancreatic and pancreatobiliary refluxes in cases with and without pancreaticobiliary maljunction: diagnosis and clinical implications. Digestion 2006;73:228-236.

-6 Csendes A, Kruse A, Funch-Jensen P, Oster MJ, Ornsholt J, Amdrop E: Pressure measurements in the biliary and pancreatic duct system in controls and patients with gallstones, previous cholecystectomy, or common bile duct stones. Gastroenterology 1979;77:1203-1210.

-7 Kamisawa T, Egawa N, Nakajima H, Okamoto A: Clinical and radiological findings in dominance of Santorini's duct. Digestion 2004;70:146-151.

8 Kamisawa T, Egawa N, Nakajima H, Matsukawa M: Dorsal pancreatic duct dominance in pancreaticobiliary maljunction. Pancreas 2005;30:e60-e63.

-9 Kamisawa T, Amemita K, Tu Y, Egawa N, Sakaki N, Tsuruta K, Okamoto A, Munakata A: Clinical significance of a long common channel. Pancreatology 2001;2:122-128.
10 Kamisawa T, Funata N, Hayashi Y, Egawa N, Nakajima H, Tsuruta K, Okamoto A, Yamaguchi T: Pathologic changes in the non-carcinomatous epithelium of the gallbladder in patients with a relatively long common channel. Gastrointest Endosc 2004;60:56-60.

11 Kamisawa T, Kuwata G, Chen PY, Tu Y, Egawa N, Nakajima H: Precancerous lesions in the gallbladder of patients with a long common channel. Dig Endosc 2006;18:192-195.

12 Shimada K, Yanagisawa J, Nakayama F: Increased lysophosphatidylcholine and pancreatic enzyme content in bile of patients with anomalous pancreaticobiliary ductal junction. Hepatology 1991;13:438-444.

$\checkmark 13$ Aoki T, Tsuchida A, Kasuya K, Endo M, Kitamura K, Koyanagi Y: Is preventive resection of the extrahepatic bile duct necessary in cases of pancreaticobiliary maljunction without dilatation of the bile duct? Jpn J Clin Oncol 2001;31:107-111.

14 Funabiki T, Matsubara T, Miyakawa S, Ishihara S: Pancreaticobiliary maljunction and carcinogenesis to biliary and pancreatic malignancy. Langenbecks Arch Surg 2009;394. 159-169.
15 Adda G, Hannoun L, Loygue J: Development of the human pancreas: variations and pathology. A tentative classification. Anat Clin 1984;5:275-283.

16 Kamisawa T, Koike M, Okamoto A: Embryology of the pancreatic duct system. Digestion 1999;60:161-165.

17 Baldwin WM: The pancreatic ducts in man, together with a study of the microscopical structure of the minor duodenal papilla. Anat Rec 1911;5:197-228.

18 Rienhoff WF, Pickrell KL: Pancreatitis. An anatomic study of the pancreatic and extrapancreatic biliary systems. Arch Surg 1945; 51:205-219.

19 Stain SC, Guthrie CR, Yellin AE, Donovan AJ: Choledochal cyst in the adult. Ann Surg 1995;222:128-133.

20 Lipsett PA, Pitt HA, Colombani PM, Boitnott JK, Cameron JL: Choledochal cyst disease. A changing pattern of presentation. Ann Surg 1994;220:644-652.

21 Raffensperger BG, Given GZ, Warner RA: Fusiform dilatation of the common bile duct with pancreatitis. J Pediatr Surg 1973;8:907910.

22 Kaneko A, Ando H, Ito T, Watanabe Y, Seo T, Harada T, Ito F: Protein plugs cause symptoms in patients with choledochal cysts. Am J Gastroenterol 1997;92:1018-1021. 